\title{
Interaction between temperature and photoperiod in regulation of flowering time in rice
}

\author{
SONG YuanLi, GAO ZhiChao \& LUAN WeiJiang* \\ Tianjin Key Laboratory of Cyto-Genetical and Molecular Regulation, College of Life Sciences, Tianjin Normal University, Tianjin 300387, \\ China
}

Received December 12, 2011; accepted February 17, 2012

\begin{abstract}
Photoperiod and temperature are two pivotal regulatory factors of plant flowering. The floral transition of plants depends on accurate measurement of changes in photoperiod and temperature. The flowering time of rice (Oryza sativa) as a facultative short-day (SD) plant is delayed under long-day (LD) and/or low temperature conditions. To elucidate the regulatory functions of photoperiod and temperature on flowering time in rice, we systematically analyzed the expression and regulation of several key genes (Hd3a, RFT1, Ehd1, Ghd7, RID1/Ehd2/OsId1, Se5) involved in the photoperiodic flowering regulatory pathway under different temperature and photoperiod treatments using a photoperiod-insensitive mutant and wild type plants. Our results indicate that the Ehd1-Hd3a/RFT1 pathway is common to and conserved in both the photoperiodic and temperature flowering regulatory pathways. Expression of $E h d 1, H d 3 a$ and $R F T 1$ is dramatically reduced at low temperature $\left(23^{\circ} \mathrm{C}\right)$, suggesting that suppression of Ehd1, Hd3a and RFTl transcription is an essential cause of delayed flowering under low temperature condition. Under LD condition, Ghd7 mRNA levels are promoted at low temperature $\left(23^{\circ} \mathrm{C}\right)$ compared with normal temperature condition $\left(28^{\circ} \mathrm{C}\right)$, suggesting low temperature and LD treatment have a synergistic role in the expression of $G h d 7$. Therefore, upregulation of $G h d 7$ might be a crucial cause of delayed flowering under low temperature condition. We also analyzed $H d l$ regulatory relationships in the photoperiodic flowering pathway, and found that $H d l$ can negatively regulate Ehdl transcription under LD condition. In addition, $H d l$ can also positively regulate $G h d 7$ transcription under LD condition, suggesting that the heading-date of rice under LD condition is also regulated by the Hdl-Ghd7-Ehd1-RFT1 pathway.
\end{abstract}

rice, photoperiod, temperature, flowering regulation, interaction

Citation: Song Y L, Gao Z C, Luan W J. Interaction between temperature and photoperiod in regulation of flowering time in rice. Sci China Life Sci, 2012, 55: 241-249, doi: 10.1007/s11427-012-4300-4

Crop yields are strongly associated with flowering time. The floral transition of crops depends mainly on the accurate measurement of changes in day length (photoperiod) and temperature, which is regulated by both endogenous genes and environmental factors. Plants can perceive and respond to changes in photoperiod [1,2]. Recent molecular biological work reveals that the rice (Oryza sativa) genes Heading date $3 a(H d 3 a)$ and Rice FT-like 1 (RFTl), orthologs of Arabidopsis FLOWERING LOCUS T (FT),

*Corresponding author (email: lwjzsq@163.com) encode florigens that can move from the leaf to the shoot apical meristem (SAM) and induce flowering in plants [3-5]. Under short-day (SD) conditions, expression of $\mathrm{Hd} 3 \mathrm{a}$ promotes rice flowering by the OsGI- Heading date $1(H d 1)-H d 3 a$ pathway which is conserved with the GIGANTEA (GI)-CONSTANS (CO)-FT pathway in Arabidopsis $[3,6,7]$. In this pathway, $H d l$ is an ortholog of $C O$ in Arabidopsis, and encodes a transcription factor with a zinc finger domain, serving as a promoter of rice flowering under SD conditions and an inhibitor under long-day (LD) 
conditions $[6,8,9]$. The circadian clock-associated protein $O s G I$ is an activator of $H d l$, upregulating the expression of $H d l$ under SD conditions, and then upregulates the expression of $\mathrm{Hd} 3 \mathrm{a}$ to finally promote flowering in rice $[7,10]$. Early heading date 1 (Ehdl), which encodes a B-type response regulator, is an upstream gene of Hd3a and RFTI and upregulates the expression of these genes to promote flowering independently of $H d l$ under SD conditions [11]. Rice Indeterminatel (RIDI)/Ehd2/Oryza sativa Indeterminate1(OsIdI) is also a positive regulator of rice flowering, and can promote flowering by upregulating Ehdl, $H d 3 a$ and RFT1 expression under both SD and LD conditions [12-14]. OsMADS51 serves as another flowering promoter that can transmit a SD promotion signal from OsGI to Ehdl, Hd3a and RFTl [15].

Under LD conditions, rice flowering is delayed, and the $O s G I-H d I-H d 3 a$ pathway is a suppression pathway. That is, $H d 1$ inhibits $H d 3 a$ transcription to delay the heading-date of rice under LD conditions [16]. Another flowering repressor Grain number, plant height, and heading date 7 (Ghd7), which encodes a transcription factor with a CCT motif, also acts to delay the flowering of rice under LD conditions by downregulating Ehdl mRNA levels [17]. This is the main suppression pathway under LD conditions. Also, Se5, encoding an important heme oxygenase enzyme involved in phytochrome chromophore biosynthesis, can inhibit the flowering time of rice under both SD and LD conditions $[18,19]$. Although rice flowering is delayed under LD conditions, it is still induced. It has been suggested that three transcription factors, RIDI/Ehd2/OsId1, OsMADS50 and $E h d 3$, are involved in rice floral induction under LD conditions. RID1/Ehd2/OsId1 and OsMADS50 can induce rice flowering by promoting Ehdl mRNA levels [12-14,20], while Ehd3, a protein with a PHD finger domain, can promote flowering under LD conditions by the downregulation of flowering repressor Ghd7 mRNA levels [21]. In addition, recent studies have shown that $H d 3 a$ and RFT1 serve different functions under different photoperiods [10,22]. $H d 3 a$ plays an important role in promoting flowering under SD conditions, while RFT1 acts as a promoter under LD conditions $[10,22]$.

Temperature also has an important effect on floral transition. Although it is well known that rice flowering is delayed under low temperature conditions, the molecular mechanisms involved in temperature response remain unknown. Our previous study investigated molecular regulation of rice flowering in different temperature conditions (27 and $23^{\circ} \mathrm{C}$ ) and revealed that $H d l$ expression was not significantly changed. However, Hd3a mRNA levels were strongly suppressed by low temperature under both SD and LD conditions [23]. This result suggested that the temperature regulatory pathway for rice flowering might interact with the photoperiod regulatory pathway. In this study, to further reveal the molecular mechanisms of temperature response in rice flowering and analyze the interaction between the temperature and photoperiod pathways, we analyzed the expression patterns of several key genes involved in the photoperiodic flowering regulatory pathway under different temperature and photoperiod treatments using Real-time PCR. Our results showed that Ehd1, Hd3a, RFT1, Ghd7 are shared and conserved in both the photoperiodic and temperature flowering regulatory pathways in rice. Several other genes, including $\mathrm{Hdl}, \mathrm{Se} 5$ and RID1/Ehd2/ OsId1, have differentiated functions in the photoperiodic and temperature regulatory pathways.

\section{Materials and methods}

\subsection{Plant materials}

Two rice materials were used in this study, Zhonghua 11 (Oryza sativa L. ssp. japonica, wild type) and lf1132, a mutant derived from a tissue culture line of Zhonghua 11. The mutant has a loss of function allele for the $H d l$ gene, with two inserted fragments and several single-base substitutions at the $H d l$ locus [23]. Plants were grown in the experimental field of the Tianjin Agricultural Academy of Science (TAAS), Tianjin Province and at Sanya, Hainan Province.

\subsection{Plant growth conditions in artificial climate cabi- nets}

Zhonghua 11 and lf1132 plants were sowed in natural fields in Tianjin, and strong seedlings were transferred at about 3 weeks old to artificial climate cabinets (MMM, Climacell, Germany). Four treatments mixing two photoperiods and two temperatures were used in the artificial climate cabinets: $\mathrm{LD}, 28^{\circ} \mathrm{C}$; LD, $23^{\circ} \mathrm{C} ; \mathrm{SD}, 28^{\circ} \mathrm{C} ; \mathrm{SD}, 23^{\circ} \mathrm{C}$. The photoperiod parameters were: LD, $14 \mathrm{~h}$ light and $10 \mathrm{~h}$ dark; SD, $10 \mathrm{~h}$ light and $14 \mathrm{~h}$ dark. The temperature parameters were: normal temperature, $28^{\circ} \mathrm{C}$; and low temperature, $23^{\circ} \mathrm{C}$.

\subsection{Real-time PCR analysis}

After $20 \mathrm{~d}$ in artificial climate cabinets, RNAs were isolated from leaves using Trizol solution (Invitrogen, USA) and treated with DNase I (NEB, USA). cDNAs were synthesized from $2 \mu \mathrm{g}$ of total RNA using M-MLV reverse transcriptase (TaKaRa, Dalian, China). One microliter of cDNA was used for real-time PCR analysis using SYBR Green PCR master mix (Tiangen, Beijing, China) and performed in a My $\mathrm{iQ}^{\mathrm{TM}} 2$ Two Color Real-Time PCR Detection System (Bio-Rad, USA). The gene-specific primers are shown in Table 1. Three replicates of each reaction were performed, and OsActin was used as an internal control for relative quantification of target gene expression. The amplification conditions were: $2 \mathrm{~min}$ at $95^{\circ} \mathrm{C}$; then 40 cycles of $30 \mathrm{~s}$ at $95^{\circ} \mathrm{C}, 30 \mathrm{~s}$ at $59^{\circ} \mathrm{C}$, and $30 \mathrm{~s}$ at $68^{\circ} \mathrm{C}$. 
Table 1 Primers used in this study

\begin{tabular}{ll}
\hline Primers & Sequences $\left(5^{\prime}-3^{\prime}\right)$ \\
\hline OsActin- $F$ & GACTCTGGTGATGGTGTCAGC \\
OsActin- $R$ & GGCTGGAAGAGGACCTCAGG \\
$H d 3 a-F$ & TTGGTAGGGTTGTGGGTGATGTGC \\
$H d 3 a-R$ & AGGTTAGGGTCACTTGGGCTTGGT \\
Ehdl-F & CGACAAAACACAAGACCACCCT \\
Ehd $1-R$ & CCTGTTTGTCTGAATCCCATCG \\
$R F T 1-F$ & TCCGAGCCCAAGCAACCCTAAC \\
$R F T 1-R$ & AGTTCCTGGTGCTGAAGTTCTG \\
Ghd7-F & AGAGGAAGAAGAGGTGCTAC \\
Ghd7- $R$ & GACATAGGTGGATGGCGGTG \\
Se $5-F$ & GGAATACTGGGTTGGAGAGATC \\
Se $5-R$ & CAGATTGCCCTCCCATTTGTAG \\
$R I D 1 /$ Ehd2/OsId $1-F$ & AACAGCAGCAGCATCACTAC \\
$R I D 1 / E h d 2 / O s I d 1-R$ & AGCAGGAGTGGTGGAGAATG \\
\hline
\end{tabular}

\section{Results}

\subsection{The heading date of mutant and wild type in dif- ferent regions}

In our previous study, we analyzed the heading-date of mutant (lf1132) and wild type (Zhonghua 11) under different photoperiods (SD and LD conditions) in detail [23]. The heading-date of the mutant was almost identical under SD and LD conditions, while the heading-date of Zhonghua 11 was $21 \mathrm{~d}$ later under LD condition than SD condition [23]. This result suggested that the mutant was photoperiod-insensitive. Here, we further investigated the heading-date of mutant and wild type at different latitudes. We sowed wild type and mutant plants in Hainan (the lower

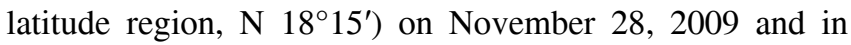
Tianjin (the higher latitude region, N $39^{\circ} 13^{\prime}$ ) on April 18, 2010. Days-to-heading of mutant and wild type were 61 and $70 \mathrm{~d}$ in Hainan, respectively, and 83 and $121 \mathrm{~d}$ in Tianjin (Figure 1A and B). The heading-date of the mutant was $9 \mathrm{~d}$ earlier than the wild type in Hainan Province. In contrast, the heading-date of the mutant was $38 \mathrm{~d}$ earlier in Tianjin (Figure 1A and B). To understand the difference in heading-date between these two areas, we further analyzed the changes in temperature and photoperiod during their growth stages in these two areas. According to the photoperiod curve, day-length changed from $10.5 \mathrm{~h}$ to $12.5 \mathrm{~h}$ during their development (from November to April) in Hainan, while day-length changed first from $12.5 \mathrm{~h}$ to $14.5 \mathrm{~h}$, and then from $14.5 \mathrm{~h}$ to $10.5 \mathrm{~h}$ (from April to October) in Tianjin (Figure 1C). This means SD conditions were dominant in Hainan, while LD conditions were dominant in Tianjin. According to the temperature curve, the average temperature was higher from sowing to heading stage (from 20 to $28^{\circ} \mathrm{C}$ ) in Hainan (Figure 1D), while the average temperature was below $22^{\circ} \mathrm{C}$ at the start of plant development (from
April to the beginning of June) in Tianjin (Figure 1E). Photoperiod and temperature results together indicate that the delayed heading-date in Tianjin is mainly caused by LD and lower temperature conditions. The heading-date of the mutant was delayed $22 \mathrm{~d}$ in Tianjin compared with Hainan, while the wild type was delayed $51 \mathrm{~d}$ (Figure 1B). These results show that the higher latitude region (lower temperature and LD conditions) strongly delays the heading-date of the wild type. The difference between the two plants may be due to higher photoperiod sensitivity in the wild type compared with the mutant.

\subsection{Hd1 regulatory relationship analysis in the photo- periodic flowering pathway}

The lf1132 mutant has a loss of function allele of the $H d l$ gene with two inserted fragments and several single-base substitutions at the $H d l$ locus, and was identified and characterized in detail in our previous study [23]. Previous studies have demonstrated that $H d l$ acts as a core regulator of the photoperiodic flowering pathway, and can directly regulate $H d 3 a$ mRNA levels to promote rice flowering under SD conditions and delay rice flowering under LD conditions $[3,16]$. Ehdl can promote rice flowering independently of $H d l$ by inducing the expression of $F T$-like genes under SD conditions [11]. RFTI and Hd3a are functionally differentiated under SD and LD conditions [22]. However, there have been no direct results to reveal the regulatory relationships between $H d l$ and several key genes involved in the photoperiodic flowering pathway such as Ehd1, RFT1, Ghd7, Se5 and RID1/Ehd2/OsId1. Here, we systemically analyzed the regulatory relationships between $H d l$ and these genes using an $H d l$-deficient mutant. Under SD condition, the Ehdl transcript displayed the same expression patterns in the wild type and the mutant; a peak before the end of the dark phase and a trough before the end of the light phase (Figure 2A). The mRNA levels of Ehdl were slightly reduced in the mutant plants (Figure 2A). However, under LD condition, the Ehdl transcript was very low in the wild-type plants, but displayed distinct expression in the mutant plants (Figure 2B), suggesting that Hdl can negatively regulate Ehdl transcription. Previous studies have shown that Ehdl can promote rice flowering independently of $H d l$ deficiency under SD conditions [11], but it appears to be regulated by $H d l$ under LD conditions.

RFT1 is a homolog of the $H d 3 a$ gene, which has been studied extensively in previous reports [3,22]. Here, RFT1 expression patterns were similar to $H d 3 a$ with a peak at the beginning of the light phase under SD condition, and its transcription level in the wild type was higher than in the mutant, indicating that $H d l$ can promote RFTl expression under SD condition (Figure 2C). Conversely, RFT1 transcription in the mutant was higher than the wild type under LD condition, indicating that Hdl can inhibit RFTl tran- 

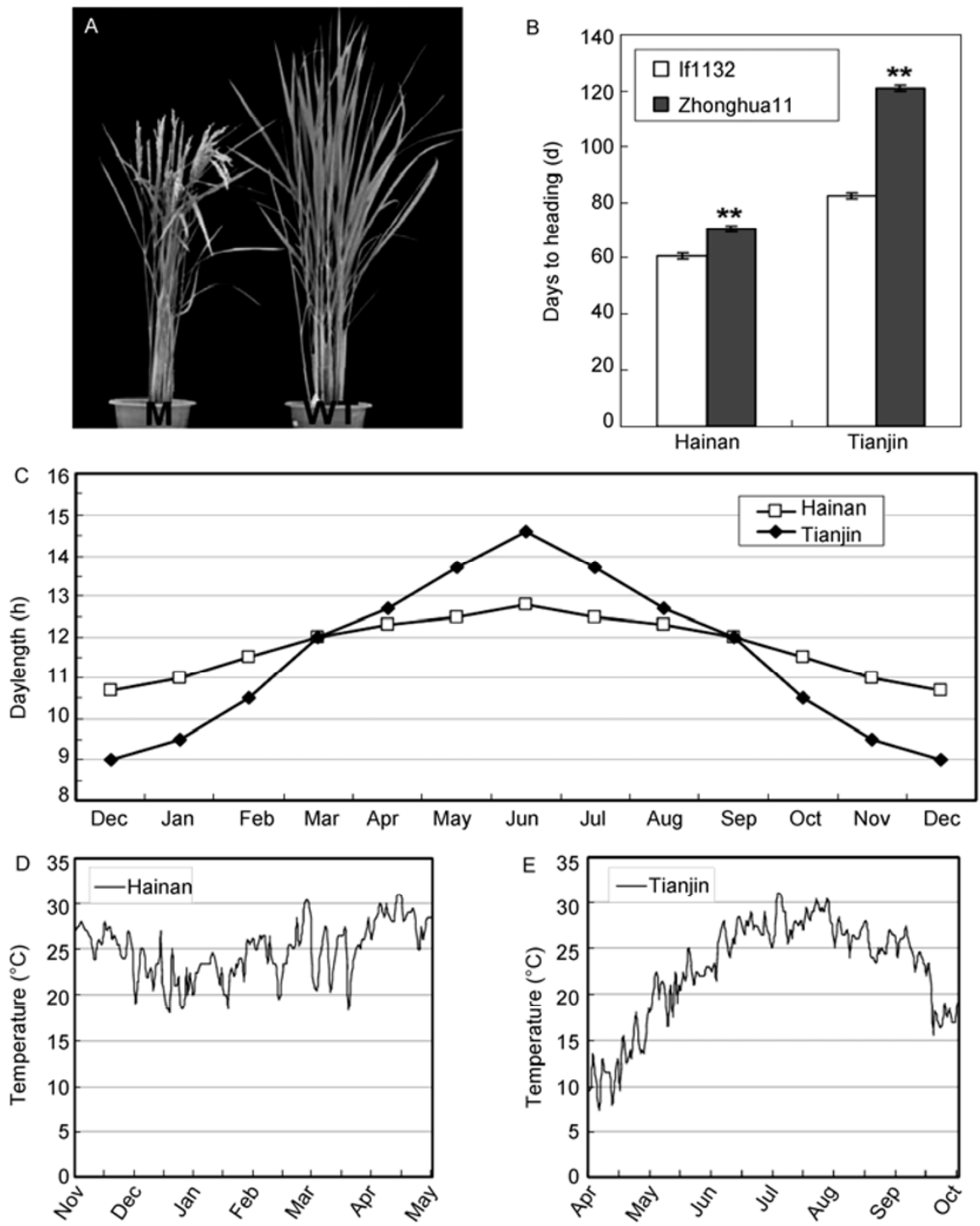

Figure 1 Analysis of heading-date in mutant and wild type plants in Hainan and Tianjin. A, The mutant displayed an early heading phenotype. M, mutant (lf1132); WT, wild type (Zhonghua11). B, The heading-date of the mutant and wild type in Hainan and Tianjin. The heading-date was recorded from 32 plants in both the mutant and wild type. C, Changes in photoperiod at Tianjin and Hainan. D and E, Changes in temperature (mean value of daily tempera-

ture) during the planting season at Hainan and Tianjin. A two-tailed Student's $t$-test was used to test the difference between two means: $* *, P<0.01$.

scription under LD condition (Figure 2D).

We also analyzed the transcription levels of $\mathrm{Se} 5$, RIDI/Ehd2/OsIdI and Ghd7, which play important roles under LD conditions, in both wild type and mutant plants. The mRNA levels of Se5 and RID1/Ehd2/OsId1 were essentially unchanged in mutant compared with in wild type (Figure 2E and F). However, the mRNA levels of Ghd7 in wild type plants were clearly higher than in the mutant, suggesting that $H d l$ can positively regulate $G h d 7$ transcription under LD conditions (Figure 2G). According to this result and previous studies, the upregulation of Ghd7 transcription by $H d l$ inhibits the transcription of Ehdl and RFT1 to further delay rice heading-date under LD condition. Therefore, the heading-date of rice under LD condition is also regulated by the $H d 1-G h d 7-E h d 1-R F T 1$ pathway.
2.3 The Ehd1-Hd3a/RFT1 pathway is common to and conserved in both the photoperiodic and temperature flowering regulatory pathways

To investigate the molecular mechanisms of the thermosensory pathway and the interaction between temperature and photoperiod in rice flowering, we measured the expression patterns of several genes involved in the photoperiodic flowering regulatory pathway in wild type and mutant plants under different temperature conditions. We first analyzed the expression of $H d 3 a$. The mRNA levels of $H d 3 a$ were reduced in both wild type and mutant plants at the low temperature $\left(23^{\circ} \mathrm{C}\right)$ compared with the normal temperature $\left(28^{\circ} \mathrm{C}\right)$ (Figure $3 \mathrm{~A}$ and $\left.\mathrm{B}\right)$, which was consistent with our previous results [23]. We then analyzed the expression of RFT1, which is the closest homolog of $H d 3 a$ 

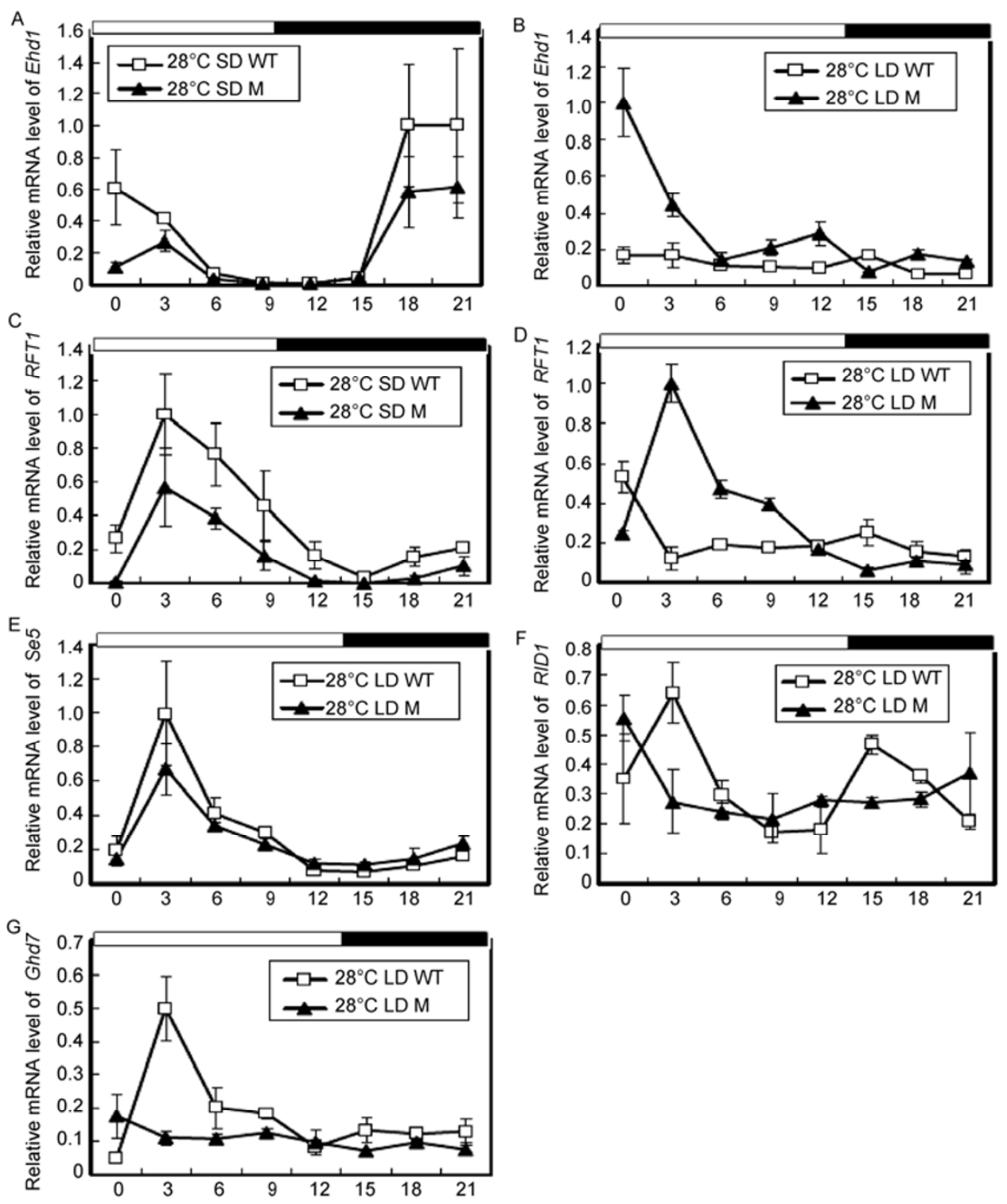

Figure 2 Ehd1, RFT1, Se5, RID1/Ehd2/OsId1 and Ghd7 expression in wild type and mutant plants under different photoperiods. Leaves were harvested from 40-day-old plants at the indicated times (once every $3 \mathrm{~h}$ for $24 \mathrm{~h}$ ) grown in artificial climate cabinets, and Real-time PCR was carried out for analysis of Ehd1, RFT1, Se5, RID1/Ehd2/OsId1 and Ghd7 expression. M, mutant (lf1132); WT, wild type (Zhonghua 11). A and B, Ehd1 expression profiles under SD and LD conditions; C and D, RFT1 expression profiles under SD and LD conditions; E-G, Se5, RID1/Ehd2/OsId1 and Ghd7 expression profiles under LD conditions.

and regulated by $H d l$ and Ehdl [22]. Under SD condition, expression of RFT1 in wild type and mutant plants was reduced at low temperature $\left(23^{\circ} \mathrm{C}\right)$ compared with normal temperature condition $\left(28^{\circ} \mathrm{C}\right)$ (Figure $3 \mathrm{C}$ and $\left.\mathrm{D}\right)$, suggesting that low temperature has an important effect on RFT1 transcription. Since $R F T 1$ is a major floral activator under LD conditions [22], we further analyzed the expression of RFT1 under LD condition. Expression of RFTI under the low temperature $\left(23^{\circ} \mathrm{C}\right)$ did not change significantly compared with the normal temperature $\left(28^{\circ} \mathrm{C}\right)$ in either wild type or mutant plants (Figure 3E and F). This may be a result of interaction between LD conditions and low temperature. On the one hand, LD conditions and low temperature suppress- es RFT1 expression to delay rice flowering time; on the other hand, RFT1 must maintain partial transcription to induce the floral transition under LD conditions. Therefore, LD conditions may have an "epistatic" effect on RFTl expression relative to the low temperature treatment.

Since RFT1 and $H d 3 a$ are probably directly regulated by Ehdl, and Ehdl plays a main role under SD conditions (Ehdl transcription was very low under LD conditions) [11], we next analyzed the expression of Ehdl at different temperatures under SD condition. The mRNA levels of Ehdl in wild type and mutant plants were dramatically reduced under low temperature condition, to the point of being almost undetectable in wild type plants at low temperature (Figure 

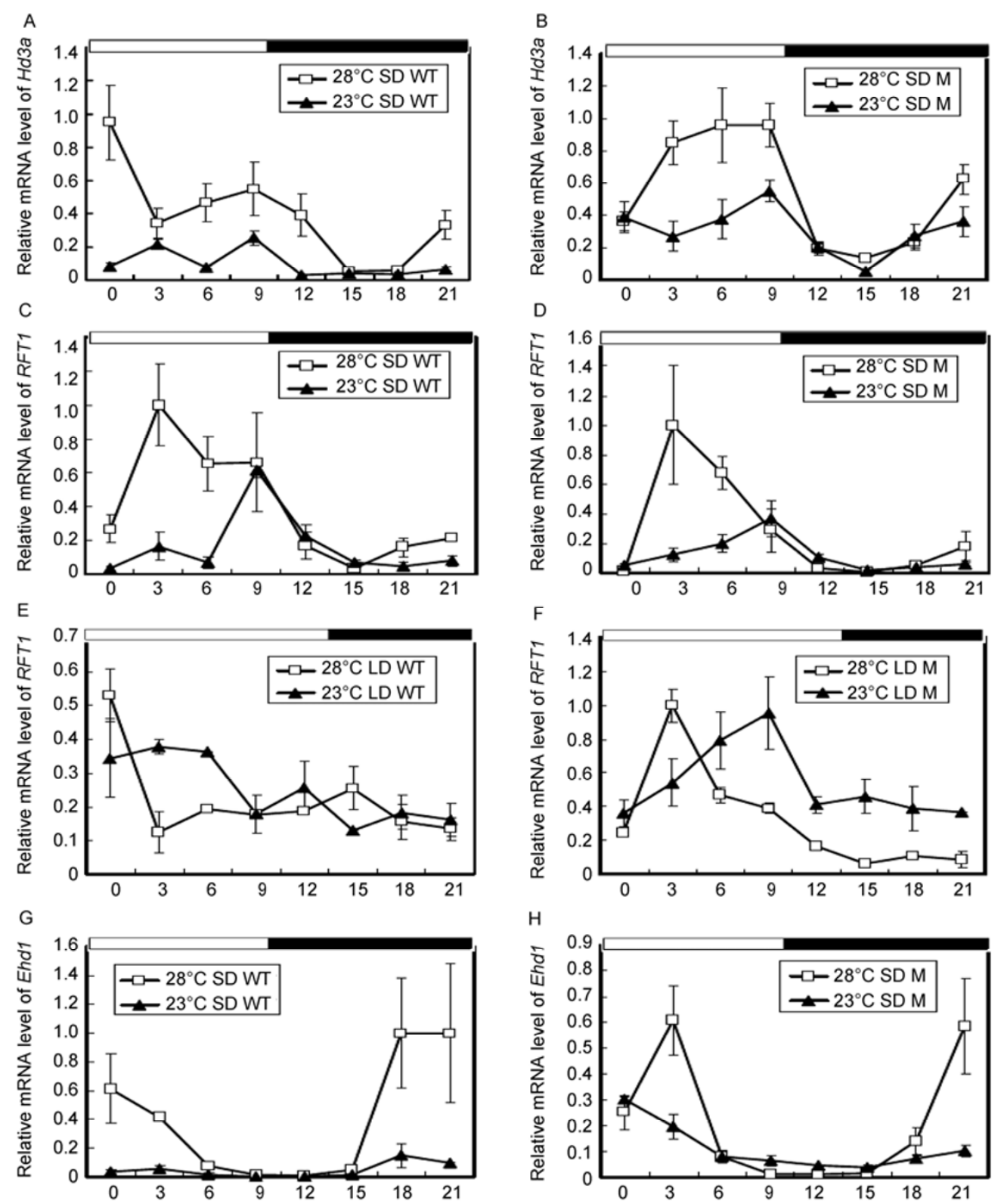

Figure $3 H d 3 a, R F T 1$ and Ehd1 expression under different photoperiods and temperatures. Leaves were harvested from 40-day-old plants at the indicated times (once every $3 \mathrm{~h}$ for $24 \mathrm{~h}$ ) grown in artificial climate cabinets, and Real-time PCR was carried out for analysis of $H d 3 a, R F T 1$ and Ehd1 expression. M, mutant (lf1132); WT, wild type (Zhonghua 11). A and B, $H d 3 a$ expression profiles at normal $\left(28^{\circ} \mathrm{C}\right)$ and low temperature $\left(23^{\circ} \mathrm{C}\right)$ under $\mathrm{SD}$ condition; A, wild type; B, mutant. C and D, RFT1 expression profiles at normal $\left(28^{\circ} \mathrm{C}\right)$ and low temperature $\left(23^{\circ} \mathrm{C}\right)$ under $\mathrm{SD}$ condition; C, wild type; $\mathrm{D}$, mutant. $\mathrm{E}$ and $\mathrm{F}$, RFT1 expression profiles at normal $\left(28^{\circ} \mathrm{C}\right)$ and low temperature $\left(23^{\circ} \mathrm{C}\right)$ under LD condition; E, wild type; F, mutant. G and $\mathrm{H}$, Ehdl expression profiles at normal $\left(28^{\circ} \mathrm{C}\right)$ and low temperature $\left(23^{\circ} \mathrm{C}\right)$ under $\mathrm{SD}$ condition; $\mathrm{G}$, wild type, $\mathrm{H}$, mutant.

3G and H). Compared with RFTl, where partial expression was maintained, Ehdl and $H d 3 a$ transcription was very low, especially in wild type plants.

Taken together, Ehd1, RFT1 and Hd3a expressions were all downregulated and suppressed under low temperature and LD conditions, and previous studies have shown that the Ehdl-Hd3a/RFTl pathway is essential in photoperiodic flowering regulation [2,3,5,10-12,22]. Therefore, we suggest that the Ehd1-Hd3a/RFT1 pathway is common to and conserved in both the photoperiodic and temperature flow- ering regulatory pathways.

\subsection{Low temperature has a significant effect on Ghd7 transcription under LD condition}

Ehd1, RFT1 and Hd3a are located downstream of the photoperiodic flowering pathway, and based on the above results, are responsive to temperature change. In addition, our previous results showed that the mRNA level of Hdl upstream of the photoperiodic flowering pathway was not sig- 
nificantly changed under low temperature condition [23]. Therefore, we analyzed the expression patterns of other genes upstream of the photoperiodic flowering under normal and low temperature conditions. We first investigated the mRNA levels of Ghd7, which plays an important role in the regulation of rice flowering time under LD conditions by downregulating Ehdl and $H d 3 a$ expression [17]. Under LD condition, Ghd7 mRNA is much more abundant at low temperature than in normal temperature condition (Figure 4A), suggesting that low temperature has a significant effect on Ghd7 transcription. This indicates that upregulation of Ghd7 expression might be a crucial cause of delayed flowering in low temperature and LD conditions. We next analyzed the expression of $G h d 7$ under SD condition. Ghd7 transcription was essentially unchanged at low temperature $\left(23^{\circ} \mathrm{C}\right)$ compared with the normal temperature $\left(28^{\circ} \mathrm{C}\right)$ (Figure 4B).

We also examined the expression of two other upstream genes, Se5 and RIDI/Ehd2/OsId1. The expression of these genes did not significantly change in different temperature treatments under either SD or LD conditions (Figure 4C-F), suggesting that $\mathrm{Se} 5$ and RIDI/Ehd2/OsId1 are not involved in the temperature flowering regulatory pathway. These results indicate that the Ghd7-Ehdl-RFT1 pathway may be important in the thermosensory response controlling rice flowering.

\section{Discussion}

Photoperiod and temperature are two important environmental factors that regulate rice flowering. Several key genes involved in the photoperiodic flowering regulatory pathway have been identified and characterized in rice, including Hd1, Hd3a, RFT1, Ehd1, Se5, RID1/Ehd2/OsId1, Ghd7, OsMADS50, DTH8/Ghd8, and Ehd3. These genes play important roles in the SD promotion, LD suppression and LD induction flowering pathways [3,5,6,10-14,17,18, 20-22,24,25]. Genes involved in temperature regulation of flowering time have been identified in Arabidopsis, but
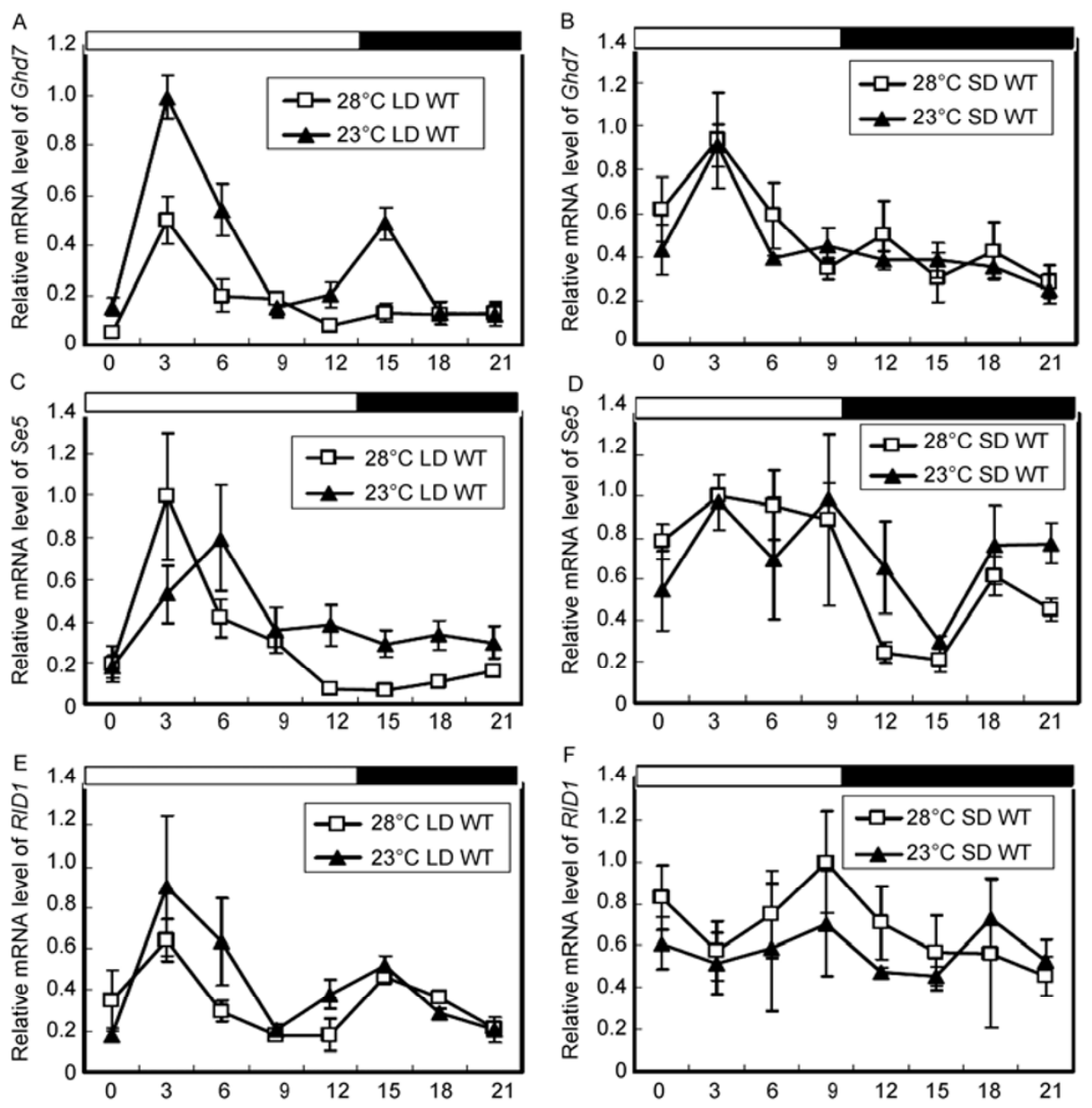

Figure 4 Ghd7, Se5 and RID1/Ehd2/OsId1 expression under different photoperiods and temperatures. Leaves were harvested at the indicated times (once every $3 \mathrm{~h}$ for $24 \mathrm{~h}$ ) from 40-day-old plants grown in artificial climate cabinets, and Real-time PCR was carried out for analysis of Ghd7, Se5 and RID1/Ehd2/OsId1 expression. WT, wild type (Zhonghua 11). A and B, Ghd7 expression profiles at normal and low temperature; A, under LD condition; B, under SD condition. C and D, Se5 expression profiles at normal and low temperature; C, under LD condition; D, under SD condition. E and F, RID1/Ehd2/OsId1 expression profiles at normal and low temperature; E, under LD condition; F, under SD condition. 
little is yet known about this process in rice. In Arabidopsis, temperature can regulate flowering time by modulating the expression of the transcription factor $F L C$ in the vernalization pathway [26-29]. The Arabidopsis SHORT VEGETATIVE PHASE (SVP) gene also plays an important role in temperature sensing by negatively regulating the expression of a floral integrator, $F T$, via direct binding to CArG motifs in the FT sequence [30], and loss of SVP function elicits insensitivity to ambient temperature changes. Recent results have also shown that nucleosomes containing the alternative histone $\mathrm{H} 2 \mathrm{~A} . \mathrm{Z}$ are essential to perceiving ambient temperature in Arabidopsis. H2A.Z can confer distinct DNA unwrapping properties on nucleosomes to modulate the transcription of temperature-responsive regulators [31]. Unlike Arabidopsis, rice can flower without a vernalization treatment and rice genes homologous to FLC have not yet been found. SVP-group MADS-box proteins mainly work as negative regulators of brassinosteroid (BR) responses in rice and have no effect on flowering time [32]. Also, whether Arabidopsis H2A.Z is an evolutionarily conserved mechanism for temperature-sensing in rice is still unknown. Therefore, more work needs to be done to reveal the molecular mechanisms of temperature control of rice flowering.

In natural fields, rice cultivars have a wide distribution from Hainan in the south of China $\left(\sim 22^{\circ} \mathrm{N}\right)$ to Heilongjiang in the north $\left(\sim 45^{\circ} \mathrm{N}\right)$, and can flower and set seeds in these local regions. There are a few distributions even in high latitude cold regions such as Heihe in the northeast of China $\left(\sim 50^{\circ} \mathrm{N}\right)$ [33]. This wide distribution and adaptation of rice may be tightly associated with the conserved Ehdl$H d 3 a / R F T 1$ pathway. RFT1 needs a balanced expression to induce to flowering in high latitude cold regions (LD and low temperature regions), that is, RFT1 transcription is reduced to delay flowering time, but must be maintained at some level to induce the floral transition under these conditions. This balance may be regulated by upstream genes such as Ghd7 and Ehdl.

Our previous results revealed that low temperature exerted a strong effect on the expression of $H d 3 a$, which is downstream of the photoperiodic regulation pathway for flowering time in rice, indicating that temperature and photoperiod might interact or crosstalk in the gene regulatory network for rice flowering [23]. Here, we further investigated the expression of several important genes involved in the photoperiodic regulatory pathway under different temperature treatments. The results indicate that the expression of Ehd1, RFTl and Ghd7 at low temperature is significantly different than under normal temperature conditions. Ehdl and RFT1 transcription is downregulated and Ghd7 transcription is upregulated under low temperature conditions, suggesting that Ehd1, RFT1 and Ghd7 are common to both the photoperiodic and temperature regulatory pathways. However, $H d l$ transcription is unchanged in different temperature treatments [23], suggesting that $H d l$ is not in- volved in the temperature flowering regulatory pathway. This result is similar to findings in Arabidopsis, where the thermosensory flowering pathway is mediated by $F T$ (a homolog of $H d 3 a$ and RFTI in rice) expression levels, while $C O$ (a homolog of $H d l$ in rice) is not essential for perceiving temperature [34]. Se5 and RID1/Ehd2/OsIdI transcriptions are also essentially unchanged in different temperature treatments. Se 5 encodes a key heme oxygenase enzyme involved in phytochrome chromophore biosynthesis [18], a component of photoperiod response. RID1/Ehd2/ OsId1 is exclusive to rice and does not have orthologous genes in Arabidopsis [12-14]. So far, no evidence exists that $S e 5$ and RIDI/Ehd2/OsIdI are involved in temperature response.

Based on our experimental results and previous studies, we would like to propose a primary model to explain the regulation of flowering time under different temperature and photoperiod conditions (Figure 5). Under SD conditions, ' $\mathrm{X}$ ' proteins strongly suppress Ehdl and its downstream $H d 3 a$ and RFT1 mRNA levels to delay rice flowering at low temperatures. So far, these upstream regulatory proteins are unknown. They may be specific to the temperature pathway. Under LD conditions, the upregulation of Ghd7 expression at low temperatures suppresses the downstream Ehdl and RFT1 mRNA levels to delay rice flowering, suggesting that the low temperature and LD treatments have a synergistic role in delaying rice flowering. It remains unclear whether unknown proteins (Ys) regulate Ghd7 or Ehdl mRNA levels in low temperature conditions.

$H d l$ and Ehdl are two crucial flowering regulators and integrators. $H d l$ has dual roles under SD and LD conditions and Ehdl plays a main role under SD conditions in the photoperiodic flowering regulatory pathway $[6,11,16]$. Our results demonstrate that $H d l$ has an important regulatory

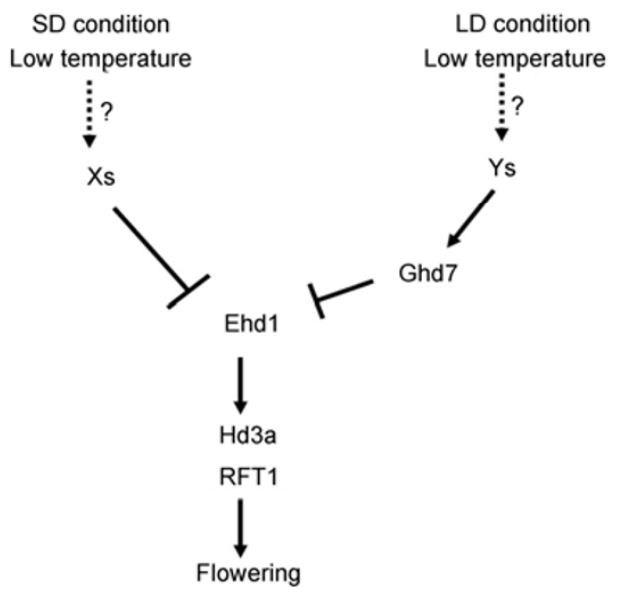

Figure 5 A model for the temperature control of flowering in rice under different photoperiod conditions. Under SD conditions, unknown proteins (Xs) may suppress Ehdl and its downstream Hd3a and RFT1 mRNA levels to delay rice flowering at low temperatures. Under LD conditions, the upregulation of $G h d 7$ expression by unknown proteins (Ys) suppresses the downstream Ehd1 and RFT1 mRNA levels to delay rice flowering. 
function on Ehdl under LD conditions. Ehdl has an important effect on rice flowering in different temperatures, suggesting that Ehdl expression is thermosensitive and involved in the temperature flowering regulatory pathway. However, transcription of $H d l$ is essentially unaffected by different temperature treatments. These results suggest that temperature and photoperiod effects have differentiated pathways for $H d l$ gene regulation. So far, the upstream regulatory genes of Ehdl (except Ghd7) are still unknown in the temperature flowering regulatory pathway, and further study is required to improve our understanding of the gene network underlying temperature regulation of rice flowering.

This work was supported by the National Natural Science Foundation of China (Grant Nos. 31171515 and 30871328), Tianjin Natural Science Foundation of China (Grant No. 11JCZDJC17900), the Program of Tianjin Municipal Education Commission (Grant No. 20090609) and Knowledge Innovation Program of Tianjin Normal University (Grant No. 52X09039).

1 Izawa T. Daylength measurements by rice plants in photoperiodic short-day flowering. Int Rev Cytol, 2007, 256: 191-222

2 Itoh $\mathrm{H}$, Nonoue $\mathrm{Y}$, Yano M, et al. A pair of floral regulators sets critical day length for $H d 3 a$ florigen expression in rice. Nat Genet, 2010, 42: 635-638

3 Kojima S, Takahashi Y, Kobayashi Y, et al. Hd3a, a rice ortholog of the Arabidopsis FT gene, promotes transition to flowering downstream of $H d 1$ under short-day conditions. Plant Cell Physiol, 2002, 43: 1096-1105

4 Corbesier L, Vincent C, Jang S, et al. FT protein movement contributes to long-distance signaling in floral induction of Arabidopsis. Science, 2007, 316: 1030-1033

5 Tamaki S, Matsuo S, Wong H L, et al. Hd3a protein is a mobile flowering signal in rice. Science, 2007, 316: 1033-1036

6 Yano M, Katayose Y, Ashikari M, et al. Hdl, a major photoperiod sensitivity quantitative trait locus in rice, is closely related to the $\mathrm{Ar}$ abidopsis flowering time gene CONSTANS. Plant Cell, 2000, 12: 2473-2483

7 Hayama R, Yokoi S, Tamaki S, et al. Adaptation of photoperiodic control pathways produces short-day flowering in rice. Nature, 2003, 422: 719-722

8 Putterill J, Robson F, Lee K, et al. The CONSTANS gene of Arabidopsis promotes flowering and encodes a protein showing similarities to zinc finger transcription factors. Cell, 1995, 80: 847-857

9 Suárez-López $\mathrm{P}$, Wheatley $\mathrm{K}$, Robson $\mathrm{F}$, et al. CONSTANS mediates between the circadian clock and the control of flowering in Arabidopsis. Nature, 2001, 410: 1116-1120

10 Komiya R, Ikegami A, Tamaki S, et al. Hd3a and RFT1 are essential for flowering in rice. Development, 2008, 135: 767-774

11 Doi K, Izawa T, Fuse T, et al. Ehdl, a B-type response regulator in rice, confers short-day promotion of flowering and controls FT-like gene expression independently of $H d l$. Genes Dev, 2004, 18: 926-936

12 Wu C, You C, Li C, et al. RID1, encoding a Cys2/His2-type zinc finger transcription factor, acts as a master switch from vegetative to floral development in rice. Proc Natl Acad Sci USA, 2008, 105: 12915-12920

13 Matsubara K, Yamanouchi U, Wang Z, et al. Ehd2, a rice ortholog of the maize INDETERMINATE1 gene, promotes flowering by upregulating Ehd1. Plant Physiol, 2008, 148: 1425-1435
14 Park S J, Kim S L, Lee S, et al. Rice indeterminate 1 (OsId1) is necessary for the expression of Ehdl (early heading date 1) regardless of photoperiod. Plant J, 2008, 56: 1018-1029

15 Kim S L, Lee S, Kim H J, et al. OsMADS51 is a short-day flowering promoter that functions upstream of Ehd1, OsMADS14, and Hd3a. Plant Physiol, 2007, 145: 1484-1494

16 Izawa $\mathrm{T}$, Oikawa $\mathrm{T}$, Sugiyama $\mathrm{N}$, et al. Phytochrome mediates the external light signal to repress $F T$ orthologs in photoperiodic flowering of rice. Genes Dev, 2002, 16: $2006-2020$

17 Xue W, Xing Y, Weng X, et al. Natural variation in Ghd7 is an important regulator of heading date and yield potential in rice. Nat Genet, 2008, 40: 761-767

18 Izawa $\mathrm{T}$, Oikawa $\mathrm{T}$, Tokutomi $\mathrm{S}$, et al. Phytochromes confer the photoperiodic control of flowering in rice (a short day plant). Plant $\mathrm{J}$, 2000, 22: 391-399

19 Andre's F, Galbraith D W, Talo'n M, et al. Analysis of PHOTOPERIOD SENSITIVITY 5 sheds light on the role of phytochromes in photoperiodic flowering in rice. Plant Physiol, 2009, 151: 681-690

20 Ryu $\mathrm{C} \mathrm{H}$, Lee $\mathrm{S}$, Cho L $\mathrm{H}$, et al. OsMADS50 and OsMADS56 function antagonistically in regulating long day (LD)-dependent flowering in rice. Plant Cell Environ, 2009, 32: 1412-1427

21 Matsubara K, Yamanouchi U, Nonoue Y, et al. Ehd3, encoding a plant homeodomain finger-containing protein, is a critical promoter of rice flowering. Plant J, 2011, 66: 603-612

22 Komiya R, Yokoi R, Shimamoto K. A gene network for long-day flowering activates $R F T 1$ encoding a mobile flowering signal in rice. Development, 2009, 136: 3443-3450

23 Luan W, Chen H, Fu Y, et al. The effect of the crosstalk between photoperiod and temperature on the heading-date in rice. PLoS ONE, 2009, 4: e5891

24 Wei X, Xu J, Guo H, et al. DTH8 suppresses flowering in rice, influencing plant height and yield potential simultaneously. Plant Physiol, 2010, 153: 1747-1758

25 Yan W, Wang P, Chen H, et al. A major QTL, Ghd8, plays pleiotropic roles in regulating grain productivity, plant height, and heading date in rice. The Molecular Plant advance online publication December 10, 2010; doi:10.1093/mp/ssq070

26 Amasino R M. Vernalization and flowering time. Curr Opin Biotech, 2005, 16: 154-158

27 Searle I, He Y H, Turck F, et al. The transcription factor FLC confers a flowering response to verbalization by repressing meristem competence and systemic signaling in Arabidopsis. Genes Dev, 2006, 20 : 898-912

28 Michaels S D, Amasino R M. FLOWERING LOCUS C encodes a novel MADS domain protein that acts as a repressor of flowering. Plant Cell, 1999, 11: 949-956

29 Helliwell C A, Wood C C, Robertson M, et al. The Arabidopsis FLC protein interacts directly in vivo with $\mathrm{SOC} 1$ and FT chromatin and is part of a high-molecular-weight protein complex. Plant J, 2006, 46: 183-192

30 Lee J H, Yoo S J, Park S H. Role of SVP in the control of flowering time by ambient temperature in Arabidopsis. Genes Dev, 2007, 21: 397-402

31 Kumar S V, Wigge P A. H2A.Z-Containing nucleosomes mediate the thermosensory response in Arabidopsis. Cell, 2010, 140: 136-147

32 Lee S, Jeong D, An G. A possible working mechanism for rice SVP-group MADS-box proteins as negative regulators of brassinosteroid responses. Plant Signal Behav, 2008, 3: 471-474

$33 \mathrm{Xu} \mathrm{W}$ X. A novel rice cultivar Heigeng 5, can be planted in high latitude and cold regions. China Rice, 1999, 3: 16

34 Balasubramanian S, Sureshkumar S, Lempe J, et al. Potent induction of Arabidopsis thaliana flowering by elevated growth temperature. PLoS Genet, 2006, 2: e106

Open Access This article is distributed under the terms of the Creative Commons Attribution License which permits any use, distribution, and reproduction in any medium, provided the original author(s) and source are credited. 